\title{
Defect studies of hydrogen-loaded nanocrystalline Gd films
}

\author{
J. Cizek ${ }^{\mathrm{a}, *}$, I. Prochazka ${ }^{\mathrm{a}}$, M. Vlach ${ }^{\mathrm{a}}$, N. Zaludova ${ }^{\mathrm{a}}$, S. Danis ${ }^{\mathrm{a}}$, G. Brauer $^{\mathrm{b}}$, W. Anwand ${ }^{\mathrm{b}}$, \\ A. Mücklich ${ }^{\mathrm{b}}, \mathrm{R}$. Gemma $^{\mathrm{c}}, \mathrm{R}$. Kirchheim ${ }^{\mathrm{c}}$, A. Pundt ${ }^{\mathrm{c}}$ \\ a Faculty of Mathematics and Physics, Charles University in Prague, V Holesovickach 2, CZ-180 00 Praha 8, Czech Republic \\ ${ }^{\mathrm{b}}$ Institut für Ionenstrahlphysik und Materialforschung, Forschungszentrum Dresden-Rossendorf, Postfach 510119, D-01314 Dresden, Germany \\ ${ }^{\mathrm{C}}$ Institut fur Materialphysik, Universität Göttingen, Friedrich-Hund-Platz 1, D-37077 Göttingen, Germany
}

\section{A R T I C L E I N F O}

\section{Article history:}

Available online 15 May 2008

\section{Keywords:}

Nanocrystalline gadolinium films Hydrogen

Slow positron implantation spectroscopy

X-ray diffraction

\begin{abstract}
A B S T R A C T
The present work reports on microstructure investigations of hydrogen-loaded nanocrystalline Gd films by means of slow positron implantation spectroscopy combined with in situ synchrotron radiation X-ray diffraction. It is found that the virgin films contain a high density of vacancy-like open volume defects at grain boundaries which trap positrons. These defects represent trapping sites also for hydrogen. With increasing hydrogen concentration the transformation from the $\alpha$-into the $\beta$-phase $\left(\mathrm{GdH}_{2}\right)$ takes place in the film. Accumulation of hydrogen at grain boundaries causes a decrease of positron localization at defects. The transformation into the $\beta$-phase is completed at $x_{\mathrm{H}} \approx 1.6 \mathrm{H} / \mathrm{Gd}$. Contrary to bulk Gd specimens, the $\gamma$-phase $\left(\mathrm{GdH}_{3}\right)$ is not formed in the nanocrystalline $\mathrm{Gd}$ films.
\end{abstract}

๑) 2008 Elsevier B.V. All rights reserved.

\section{Introduction}

The $\mathrm{Gd}-\mathrm{H}$ system attracts attention of researchers because (i) it can absorb a large amount of hydrogen and exhibits a negative enthalpy of solution for atomic hydrogen [1], (ii) thin Gd films can be reversibly switched from optically refractive to transparent state by exposure to hydrogen [2]. The knowledge about the $\mathrm{Gd}-\mathrm{H}$ phase diagram is yet incomplete, especially at low temperatures [1]. With increasing hydrogen concentration (expressed as atom ratio $\mathrm{H} / \mathrm{Gd}$ throughout this paper) bulk Gd undergoes the following phase transition sequence: $\alpha$-phase (hcp, GdH) $\rightarrow \beta$-phase (fcc, $\left.\mathrm{GdH}_{2}\right) \rightarrow \gamma$-phase (hcp, $\mathrm{GdH}_{3}$ ). In the $\alpha$-phase at room temperature, hydrogen solubility is very low $\left(x_{\mathrm{H}}<0.01\right)$. Under normal conditions, Gd samples always contain some traces of the $\beta$-phase. With increasing hydrogen concentration, the volume fraction of the $\beta$-phase increases at the expense of the $\alpha$-phase. At $x_{\mathrm{H}} \approx 1.9$, the bulk sample is completely transformed into the $\beta$-phase. Formation of the $\gamma$-phase begins place from $x_{\mathrm{H}} \approx 2.3$ and is completed at $\approx 2.8$.

Hydrogen-loaded thin films often behave very differently from the corresponding bulk materials [3]. It happens because of two main reasons: (i) contrary to free standing bulk samples which

\footnotetext{
* Corresponding author at: Department of Low-Temperature Physics, Faculty of Mathematics and Physics, Charles University, V Holesovickach 2, CZ-180 00 Praha 8, Czech Republic. Tel.: +420 2 21912788; fax: +420221912567.

E-mail address: jakub.cizek@mff.cuni.cz (J. Cizek).
}

exhibit isotropic lattice expansion when loaded with hydrogen, thin films are clamped to a substrate. The clamping prevents the in-plane lattice expansion and causes appearance of large in-plane compressive stresses (in the GPa range) [4], which influence the behavior of the loaded films. (ii) Thin films, and in particular nanocrystalline films, often exhibit a high density of lattice defects. It is known that $\mathrm{H}$ strongly interacts with defects [2]. Thus, the defect structure is an important factor which must be considered in investigations of hydrogen-loaded specimens.

Nanocrystalline Gd films loaded with hydrogen were studied in the present work. Defect studies of the films were performed by slow positron implantation spectroscopy (SPIS) utilizing monoenergetic slow-positron beam with adjustable energy. SPIS was combined with in situ X-ray diffraction (XRD) studies.

\section{Experimental details}

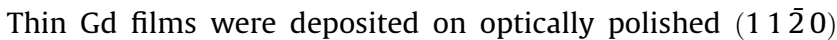
sapphire substrates by cold cathode beam sputtering in an UHV chamber $\left(10^{-10} \mathrm{mbar}\right)$. As determined by transmission electron microscopy (TEM), film thickness was $1240 \mathrm{~nm}$ and films exhibited elongated column-like grains with a typical lateral width of $\approx 50 \mathrm{~nm}$. The film surface was subsequently covered with a $30 \mathrm{~nm}$ thick Pd cap at room temperature in order to prevent oxidation, and to facilitate hydrogen absorption into the Gd layer [5]. The virgin films were firstly anodized at a constant potential of $0.8 \mathrm{~V}$ in order to remove hydrogen dissolved already in the as-deposited specimens. Subsequently, the films were step-by-step cathodically 
charged with hydrogen [6] in a galvanic cell filled with $1 \mathrm{M} \mathrm{KOH}$ electrolyte. The hydrogen concentration in the sample was calculated from the Faraday's Law. Bulk Gd (99.9\%) samples, annealed at $700{ }^{\circ} \mathrm{C}$ for $1 \mathrm{~h}$ in vacuum $\left(10^{-8} \mathrm{mbar}\right)$, were used as reference samples for SPIS investigations.

The SPIS studies of thin films were performed at the magnetically guided positron beam facility "SPONSOR" [7] with a positron energy adjustable from 0.03 to $36 \mathrm{keV}$. Doppler broadening of the annihilation line was measured by an HPGe detector with an energy resolution of $(1.09 \pm 0.01) \mathrm{keV}$ at $511 \mathrm{keV}$ and characterized in terms of the $S$ parameter. An energy window of $744 \mathrm{eV}$ centered at $511 \mathrm{keV}$ was used for evaluation of the $S$ parameters. The in situ XRD investigations of hydrogen-loaded films were performed at HASYLAB (DESY, Hamburg) using synchrotron radiation with a wavelength $\lambda \approx 0.5 \AA$.

\section{Results and discussion}

The dependence of the $S$ parameter on positron energy $E$ measured on the reference bulk Gd samples is plotted in Fig. 1A. Two bulk samples were compared: sample A was annealed at vacuum and its surface was subsequently covered with the Pd over-layer; sample B was also annealed at vacuum but its surface was not covered by Pd. For sample A, the $S$-values converge at high energies to a bulk value $S_{0}=0.5078(5)$. All the $S$ parameters measured in this work were normalized to this value $S_{0}$. It can be seen from Fig. 1A that $S$ parameters measured at higher energies for the sample $B$ reasonably converge the bulk value, too. From fitting of the $S(E)$ curves by VEPFIT [8] (solid lines in Fig. 1A) we obtained the bulk positron diffusion length $L_{+, G d}=(270 \pm 30)$ and $(280 \pm 20) \mathrm{nm}$ for the samples A and B, respectively. These diffusion lengths indicate that both reference $\mathrm{Gd}$ samples are well annealed and their bulk can be considered as a defect-free material. However, the structure of the subsurface region differs considerably in both reference samples. Sample A exhibits a drop of $S$ at low energies due to positron annihilations inside the Pd cap. At higher energies, there is an increase of $S$ caused by an increasing contribution of positron annihilations in Gd. The data indicate a broad maximum of $S$ around $12 \mathrm{keV}$, which seems to be reflected also by VEPFIT results below. Subsequently, $S$ decreases slightly to the bulk value. Thus, going from the surface into the depth, one can consider three regions in the sample A: (i) the Pd cap, (ii) a Gd subsurface layer, which exhibits slightly enhanced $S$ parameter most probably due to damage caused by sputtering of the Pd cap, and (iii) a defect-free bulk Gd region. From fitting of the $S(E)$ curve it was found that the width of the layer (ii) is $(120 \pm 20) \mathrm{nm}$, and this layer exhibits a positron diffusion length of
$(100 \pm 10) \mathrm{nm}$. The Pd layer (i) is highly defected which is testified by a low positron diffusion length of $(20 \pm 5) \mathrm{nm}$, its width was fixed at $20 \mathrm{~nm}$ known from TEM. The $S(E)$ curve for the reference sample B exhibits a maximum at $7 \mathrm{keV}$ due to positron annihilations inside the Gd oxide layer formed on the uncovered Gd surface exposed to air. Subsequently, $S$ converges to the bulk value. Thus, the sample B consists of two layers only: (i) a Gd oxide layer and (ii) the bulk Gd region. The width of the oxide layer of $(240 \pm 20) \mathrm{nm}$ was obtained from a fit of the $S(E)$ curve using this model. The positron diffusion length in the oxide layer is estimated to be $(110 \pm 10) \mathrm{nm}$.

The $S(E)$ curve for the virgin (unloaded) Gd film is plotted in Fig. 1B. The drop of $S$ at low energies is due to positron annihilations inside the Pd cap. An increasing fraction of positrons annihilating inside the Gd layer causes an increase of $S$ with increasing positron energy $E$. In the energy range from 5 to $11 \mathrm{keV}$ almost all positrons annihilate inside the Gd layer, and $S$ reaches a plateau-like value. Finally, at higher energies positrons begin to penetrate also into the sapphire substrate and this contribution causes a decrease of $S$. From comparison with the reference bulk samples it becomes clear that the Gd film exhibits a substantially higher $S$ value. This is a consequence of nanoscale grain size, which leads to a significant volume fraction of grain boundaries and, thereby, a significant contribution of positrons trapped at open volume defects at grain boundaries. The trapped positrons raise the $S$ parameter and decrease the positron diffusion length in the Gd layer. The $S(E)$ curves for Gd films were fitted by VEPFIT using a three-layer model: (i) Pd cap, (ii) Gd layer, and (iii) sapphire substrate. For the Pd cap we obtained practically the same results as those for Pd cap deposited on the reference sample A. The Gd layer exhibits the positron diffusion length of $(168 \pm 3) \mathrm{nm}$, i.e. shorter than that in defect-free Gd, due to positron trapping at grain boundaries. The sapphire substrate exhibits a rather short positron diffusion length of $(38 \pm 3) \mathrm{nm}$ most probably due to positron trapping at dislocations introduced by polishing of the substrate surface.

The $S(E)$ curves measured on the films loaded to various hydrogen concentrations are plotted in Fig. 1B. Clearly, hydrogenation of the film causes a strong decrease of the $S$ parameter for the Gd layer. The $S$ parameters and positron diffusion lengths for the Gd layer obtained from fits of the $S(E)$ curves are plotted in Fig. $2 \mathrm{~A}$ as a function of hydrogen concentration.

The XRD spectrum of the unloaded virgin film does not only contain reflections corresponding to the $\alpha$-phase but also weak reflections which can be attributed to the $\beta$-phase. Thus, traces of the $\beta$-phase are present already in the unloaded virgin film. The out-of-plane lattice parameters of the $\alpha$ and the $\beta$-phase obtained
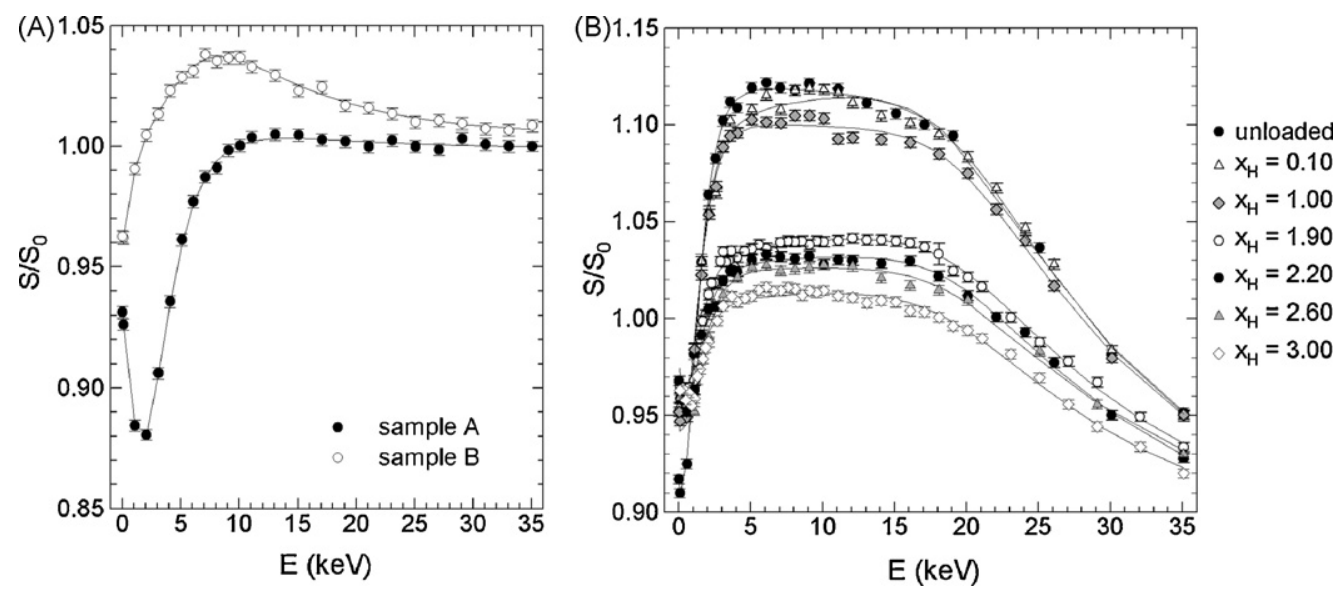

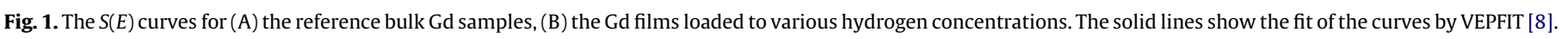



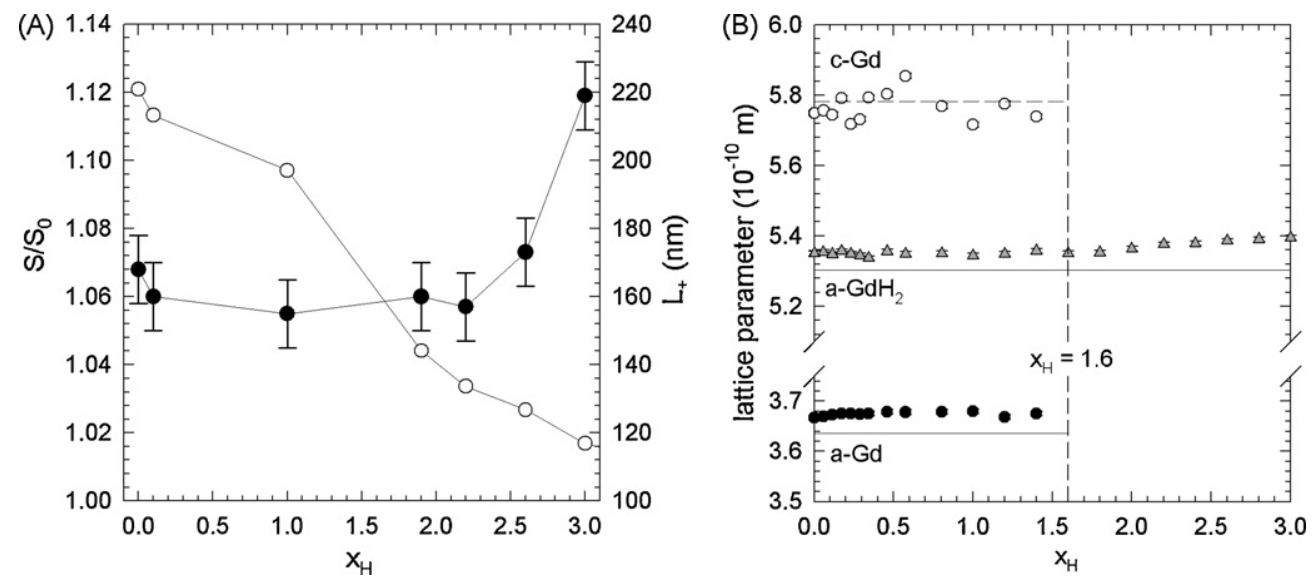

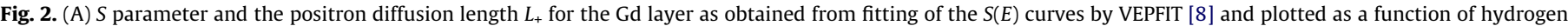

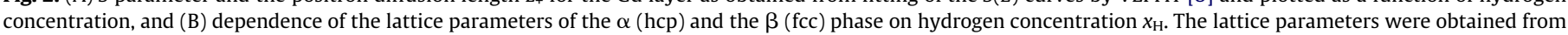
fitting of the XRD spectra. Solid lines show the lattice parameters for the bulk Gd.

from fitting of the XRD spectra are plotted in Fig. $2 \mathrm{~B}$ as a function of hydrogen concentration. The lattice parameters known from the literature for bulk Gd [9] and $\mathrm{GdH}_{2}$ [10] are shown in Fig. 2B by solid lines. The out-of-plane lattice parameter $a$ measured in the film is higher than in bulk Gd. It testifies compressive in-plane stresses in the film. The $c$ parameter is almost the same as that for bulk Gd. It is in concordance with texture measurements which revealed that

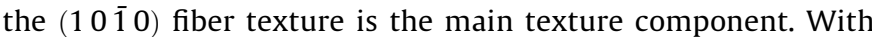
increasing hydrogen concentration, the $\alpha$-phase transforms into the $\beta$-phase. It is seen as a gradual decrease of the intensity of the $\alpha$-phase reflections while that of the $\beta$-phase reflections grows up. The lattice parameters of both phases remain approximately constant during the phase transition. At $x_{\mathrm{H}} \approx 1.6$, the transformation is completed and the film contains only the $\beta$-phase. Thus, the two-phase field $(\alpha+\beta)$ in the nanocrystalline Gd film is narrower compared to bulk Gd. Such narrowing was observed in a number of nanocrystalline metals [3]. A further increase of the hydrogen content causes only a broadening of the $\beta$-phase reflections and an expansion of the lattice seen as an increase of the lattice parameter $a$. Thus, one can conclude that the $\gamma$-phase is not formed in the nanocrystalline Gd film. Most probably for the nanocrystalline structure it is energetically more favorable to stay in the $\beta$-phase. The increase of the hydrogen concentration makes the film structure less defined (i.e. local variations of the lattice constant become larger) which is seen by a broadening of the diffraction profiles.

From the comparison of SPIS and XRD results we can conclude that the strong decrease of the $S$ parameter for the Gd layer observed in the hydrogenated film occurs mainly due to transition from the $\alpha$-phase into the $\beta$-phase. In addition, hydrogen fills the open volume defects and grain boundaries which exhibit a more open structure. It lowers the ability of defects to trap positrons and increases the local electron density at defects. Both effects contribute to a lowering of $S$ and an increase of the positron diffusion length in the Gd layer.

\section{Conclusions}

Nanocrystalline Gd films with thickness of $1240 \mathrm{~nm}$ were deposited on sapphire substrate and step-by-step loaded with hydrogen. SPIS studies show a strong decrease of the $S$ parameter for the $\mathrm{Gd}$ layer caused by $\alpha$ to $\beta$ phase transition and hydrogenation of grain boundaries. Traces of the $\beta$ phase are found to be present already in the virgin film. The two-phase field $(\alpha+\beta)$ is found to be narrower compared to bulk Gd. The $\gamma$-phase is found to not form at high hydrogen concentrations.

\section{Acknowledgements}

Financial support from The Czech Science Foundation (project No. 202/05/0074), The Ministry of Education, Youth and Sports of The Czech Republic (project No. MS 0021620834) and The Alexander von Humboldt Foundation is highly acknowledged.

\section{References}

[1] F.B. Manchster (Ed.), Phase Diagrams of Binary Hydrogen Alloys,, ASM International, Materials Park, 2000, p. 58.

[2] J.N. Huiberts, R. Griessen, J.H. Rector, R.J. Wijngaarden, J.P. Dekker, D.G. de Groot, N.J. Koeman, Nature 380 (1996) 231.

[3] A. Pundt, R. Kirchheim, Annu. Rev. Mater. Res. 36 (2006) 55.

[4] U. Laudahn, A. Pundt, M. Bicker, U.V. Hülsen, U. Geyer, T. Wagner, R. Kirchheim, J. Alloys Compd. 293-295 (1999) 490.

[5] M.A. Pick, J.W. Davenport, M. Strongin, G.J. Dienes, Phys. Rev. Lett. 43 (1979) 286.

[6] R. Kircheim, Prog. Mat. Sci. 32 (1988) 261.

[7] W. Anwand, H.-R. Kissener, G. Brauer, Acta Phys. Polonica A 88 (1995) 7.

[8] A. van Veen, H. Schut, M. Clement, J. de Nijs, A. Kruseman, M. Ijpma, Appl. Surf. Sci. 85 (1995) 216.

[9] F.J. Darnell, Phys. Rev. 130 (1963) 1825.

[10] M. Ellner, H. Reule, E.J. Mittemeijer, J. Alloys Compd. 279 (1998) 179. 